\title{
Spinal cord damage and operations for coarctation of the aorta: aetiology, practice, and prospects
}

\begin{abstract}
An inquiry was made into the clinical practice and paraplegia rate associated with operations for coarctation of the aorta conducted by surgeons in the United Kingdom and Ireland. Paraplegia occurred in 16 patients in a total of 5492 operations, an incidence of $0.3 \%$, or once in 343 operations. The aetiology and measures that may be taken to avoid this complication are discussed.The medicolegal consequences need no emphasis and it is recommended that finger palpation alone as a method of assessing the adequacy of distal aortic pressure should be abandoned in favour of continuous electromanometric visual display of the aortic pressure in all patients. In the long term, serious consideration must be given to developing spinal cord monitoring using somatosensory evoked potentials.
\end{abstract}

One of the greatest tragedies that can befall an active child is the development of severe and disabling neurological damage after an apparently straightforward operative procedure. The consequent deprivation and anguish to the patient, family, and surgeon, to say nothing of probable litigation, makes this a subject worthy of serious consideration.

The potential for damage to the spinal cord arises on account of its variable, disputed, and sometimes precarious blood supply. The spinal cord is at risk not only during operations on the thoracic aorta but also in orthopaedic and neurosurgical operations on the thoracic spine, particularly those for the mechanical correction of scoliosis. Although the risk of paraplegia in operations for traumatic rupture of the aorta and of acute dissection is high (indeed, in these conditions the patient may present with paraplegia), the incidence after operations for coarctation of the aorta is fortunately low. Nevertheless, since many operations for these conditions are undertaken worldwide, there is a group of patients suffering consequential paraplegia; and because of the understandable reluctance of surgeons to publish these tragedies, this number is probably considerably underestimated. ${ }^{1}$

The blood supply of the spinal cord is derived from the anterior spinal artery, which provides a rich anastomotic network. This artery is supplied by branches of the vertebral arteries, radicular branches from each of the intercostal arteries, and other collaterals.

Address for reprint requests: Mr G Keen, Bristol Royal Infirmary, Bristol BS2 8HW.
Whereas in most individuals the anterior spinal artery is a continuous vessel, unhappily it is occasionally discontinuous. Since much of the blood supply to the anterior spinal artery arises from the cervical vessels and lower thoracic and upper lumbar vessels (the latter through the great radicular arteries, particularly of Adamkiewicz ${ }^{2}$ ), possibly clamping of the aorta or of intercostal vessels for operative procedures may interfere appreciably with the blood supply to an isolated segment of the thoracic spinal cord, with consequent ischaemic transection.

A recent study of the vascular anatomy of the lower spinal cord has thrown interesting light on the aetiology of paraplegia in man. Although the use of distal shunting after aortic cross clamping almost invariably protects from paraplegia, some cases of paraplegia are reported despite these measures. Svenson and his colleagues in $1986^{3}$ studied the haemodynamics, paraplegia rate, and spinal cord blood flow with radioactive microspheres in adult baboons, with particular reference to the arteria radicularis magna (the main lumbar arterial branch to the lower spinal cord). There were no significant left ventricular haemodynamic advantages with shunting, but shunting significantly increased lumbar spinal cord blood flow, which correlated with the distal aortic mean pressure. Lower thoracic spinal cord blood flow did not, however, increase during shunting and did not correlate with the distal aortic pressure. This is due to the vascular anatomy of the anterior spinal artery, which, as in man, was much smaller above than below the entry of the arteria radicularis magna. ${ }^{4}$ Resistance to flow, as calculated by Poiseuille's equation, is 11 
times greater in man up the anterior spinal artery than down this artery. These findings show that distal perfusion of the aorta using shunts after cross clamping may provide adequate lumbar spinal cord protection, but the arrangement of the vascular anatomy so described leaves the lower thoracic spinal cord at risk from ischaemia. They concluded that during thoracic aortic cross clamping without distal perfusion the lumbar spinal cord is the area most sensitive to ischaemia. A distal shunt, however, increases lumbar spinal cord blood flow, but does not alter the protection of thoracic spinal cord blood flow during prolonged thoracic aortic cross clamping, thus explaining some cases of paraplegia following an apparently well conducted operation for coarctation of the aorta even when adequate distal shunting is used.

Wadouh and his colleagues undertook direct measurements of the oxygen tension on the surface of the spinal cord of pigs after occlusion of the descending aorta in a serious of elegant experiments, and showed that oxygen tension decreased dramatically after this procedure, and that the decrease was time related. Furthermore, the production of paraplegia in these animals could be predicted at certain levels of surface oxygen tension, and these changes were directly related to the low blood pressures recorded distal to the aortic cross clamp. ${ }^{5}$

Operations on the descending thoracic aorta are indicated for acute traumatic rupture, for some acute dissections, and for chronic aneurysms, but a common indication is for the resection and anastomosis of coarctation of the aorta, a congenital abnormality.

These operations are complicated by the need to cross clamp the aorta high up and at the same time prevent left ventricular strain and to protect the kidneys, spinal cord, and abdominal viscera from the effects of ischaemia.

\section{Paraplegia and surgery for coarctation}

The adequacy of blood supply to the lower spinal cord after cross clamping should depend logically on the number of intercostal vessels that are sacrificed and on the duration of aortic cross clamping. At first sight it would appear that the more intercostal arteries are divided or the longer the period of aortic cross clamping the greater is the risk to the patient of developing paraplegia. This, however, is not necessarily the case and the conclusions of Brewer are important. ${ }^{1}$ This review was summarised as follows:

1 An incidence of $0.41 \%$ of spinal cord complications in 12532 patients who had surgical correction of coarctation of the aorta was found by the author in a survey completed by leading thoracic surgeons (one case in 250 operations).

2 Twenty three additional cases with and without surgery were collected from published reports.

3 There were eight cases of spontaneous spinal cord injury without operation, six collected from publishec reports and two from his own series.

4 The concept of the segmental blood supply to the cord by multiple consistent radicular branches from the intercostal vessels is erroneous.

5 Neither sacrifice of intercostal arteries no $\overrightarrow{0}$ duration of occlusion of the aorta was related to spi $=$ nal cord injuries in all cases reported in this series.

6 Although the extent of the collateral circulation may be estimated preoperatively, the sufficiency of this circulation must be verified at operation. The most reliable test is the accurate measurement of the aortic pressure above and below the coarctation before and after occlusion of the coarctation. This test should be added to the techniques that alt surgeons use in repairing coarctation of the aorta. 7 The surgeon must also be prepared at every oper ation for coarctation of the aorta to provide addi tional protection of the spinal cord in patients with proved poor collateral circulation by one of threev means: hypothermia, left heart bypass, or jumpi grafts.

8 The variation of the blood supply to the anterio spinal artery may not permit even the briefest cros clamping, despite the use of the above mentioned pro $\frac{\mathbb{Q}}{2}$ tective measures.

9 Since this may occur in any case and is beyond the control of the surgeon, it is essential that this is made known to the patient before the operation.

\section{Determination of adequacy of distal aortic perfusion during operations for coarctation of aorta}

After aortic cross clamping it is necessary to have at accurate assessment of the adequacy of blood supply? to the lower limbs, abdominal viscera, kidneys, and especially the spinal cord. In coarctation of the aorta윽 when there is a completely obstructing diaphragm cross clamping of the aorta above and below this diaphragm should cause no change in distal aortion perfusion, for adequate collateral circulation to the lower half of the body is provided through the hyper trophied internal mammary, parascapular, and otherw arteries, which return blood in a retrograde manne? into the lower aorta via the intercostal arteries. Im most cases, however, the coarctation is not a completed diaphragm and there may be appreciable blood flow through the narrowed segment. In these cases aortic cross clamping will deprive the lower aortic segmen? of a varying amount of blood, depending on the degree of narrowing produced by the coarctation. In those patients with a less than tight coarctation and 
with appreciable blood flow through the coarctation there will be other indications that this is so. In these cases the femoral pulses may be palpable, although delayed, and there may be less than the usually readily palpable collaterals over the posterior chest wall. Further, upper limb hypertension may be modest or absent. In this group of patients special care is required to monitor the lower aortic segment after cross clamping.

Experienced cardiothoracic surgeons can assess fairly adequately the distal aortic pressure after aortic cross clamping by digital palpation of the distal aorta. The educated finger can certainly distinguish between a soft and a tense aorta, but many surgeons claim (without much evidence) that digital palpation helps them to assess this pressure to within 10 or $15 \mathrm{~mm} \mathrm{Hg}$. Undoubtedly the pressure in the distal aorta is measured more accurately by an indwelling needle in the distal aorta or by means of a femoral artery catheter attached to an electromanometric device giving continuous visual display of the arterial pressure. ${ }^{6}$

\section{Past and present surgical practice}

In March 1985 I corresponded with those 90 members of the Society of Thoracic and Cardiovascular Surgeons of Great Britain and Ireland whom I knew to have personal experience of operations for coarctation of the aorta. My interest arose from being concerned with the legal actions of several patients who had suffered paraplegia after coarctation operations by other surgeons. In these cases inquiry had been made by the plaintiffs about the method of monitoring distal aortic perfusion after aortic cross clamping.

In my questionnaire I asked which method they routinely used - whether it was digital palpation or whether it was by electromanometric measurements. I was interested to know what their practice was in 1985 and furthermore what had been their practice in 1980 as I was attempting to determine what might reasonably be considered to be usual and therefore prudent practice at that time. I also inquired into the incidence of paraplegia after the operation.

I received 74 completed questionnaires from the 90 surgeons, and an analysis of these has proved interesting, although inconclusive. Forty two surgeons replied that in 1980 they did not use electromanometric measurements in the distal aorta after aortic cross clamping, but that they relied upon digital palpation of the lower aortic segment to give information on the adequacy or otherwise of distal perfusion.

Twenty seven surgeons routinely measured distal aortic pressures after aortic cross clamping; a further five did not do so regularly, but did use monitoring when digital palpation of the distal aorta suggested an inadequate blood supply. Since therefore the majority clearly did not use electromanometric measurement in 1980 , failure to do so could not be regarded as unusual or unacceptable practice at that time.

In 198535 of this same group of surgeons did not measure distal aortic pressure electromanometrically and continued to rely on digital palpation, but 39 reported that they now invariably measured distal aortic pressures. Thus 12 surgeons who did not routinely measure distal aortic pressures in 1980 were doing so in 1985.

Neither method of distal aortic pressure assessment was especially preferred by any particular age group of surgeons, although the digital method of palpation of aortic pressure was used frequently by those surgeons with very great experience. No surgeon measured distal aortic pressure while operating on coarctation of the aorta in the first few weeks of life, as this is not practical and cross clamp times are very short.

\section{Incidence of spinal cord damage}

In this inquiry 74 surgeons reported a total of 5492 operations for coarctation of the aorta. Among these, there were 16 patients with consequent paraplegia, an incidence of $0.3 \%$ or one in 343 operations. Fourteen of these followed primary operations, one occurring suddenly and permanently one month after operation. The remaining two followed second or third operations for coarctation, and in neither case was any form of bypass used.

A further three patients were reported to have had postoperative temporary paraplegia, all of whom recovered completely within 10 days of operation. A few surgeons separately reported that several of their patients had developed an unusual gait after operation, which may have been due to a mild cauda equina lesion. Therefore in addition to 16 patients with permanent paraplegia three sustained temporary paraplegia and an unknown number of other patients may have developed less obvious or transient lesions of the spinal cord or cauda equina.

\section{Somatosensory evoked potentials}

A most attractive method of assessing spinal cord function during these operations is by the continuous monitoring of spinal cord function by means of somatosensory evoked potentials. The stimulation of various sensory systems will produce a signal identifiable by an electroencephalogram on the relevant part of the surface of the brain provided that the transmitting nerve is intact. This has been understood for many years in ophthalmic surgery, where the 
flashing of a light before the eye is picked up as a signal at the occipital cortex provided that the optic nerve and other tracts are intact. Similarly, stimulation of peroneal or tibial nerves will produce a continuous signal on the cerebral cortex provided that the spinal cord is intact. This simple concept is unfortunately much more difficult to transfer to clinical practice than superficial consideration might suggest. The equipment used is specialised and interpretation of the signals produced are best supervised by neurophysiologists and trained technicians in the operating theatre. Experience has shown that once the technique has been learned a trained member of the team, usually the anaesthetist, will be able to interpret normal and abnormal cerebral responses to peripheral stimulation during surgery.

\section{Spinal cord monitoring}

Laschinger and his colleagures ${ }^{78}$ undertook an investigation of the experimental and clinical assessment of the adequacy of partial bypass in the maintenance of spinal cord blood flow during operations on the thoracic aorta using spinal cord impulse conduction (somatosensory evoked potentials). They concluded that maintenance of a distal aortic pressure greater than $60-80 \mathrm{~mm} \mathrm{Hg}$ will uniformly preserve spinal blood flow in the absence of critical intercostal exclusion. Should distal aortic pressure be inadequate, early reversible changes in the somatosensory evoked potentials will alert the surgeon; and failure to institute measures to reverse these changes may result in paraplegia.

With the availability of these methods many centres (commonly in the United States and two or three in the United Kingdom) are modifying their techniques and use somatosensory evoked potentials. Inquiry at these centres shows that a prolonged learning process is required before the interpretation of data is of sufficient accuracy for this to become a clinically reliable technique, and consequently it must be regarded as an experimental technique in the hands of learner groups. Although this appears to be a formidable problem, it is clear that we have a duty to develop these techniques and to transmit them to the operating theatre, for surgery of the thoracic aorta and for scoliosis. The initial and revenue costs of such a system are high, and so is the cost of training appropriate personnel; but the equipment and personnel in any one city could be made available to help those surgeons or neurologists who might from time to time need this assistance. It has, of course, yet to be proved that in the present state of the art the use of somatosensory evoked potentials offers greater reliability than the monitoring of distal aortic pressure. Pollock and his colleagues in Glasgow, who are evaluating somatosensory evoked potential monitoring, believe that their initial experience is promising and that itso intraoperative use can identify quickly the patient ato risk of ischaemic cord damage and allow alternative음 repair methods, avoiding cross clamping, to be used. $\bar{s}$ Although they believe that the technique holds great ${ }_{\circ}^{\circ}$ promise they are of the opinion that much works remains to be done before an appropriate degree of ${ }_{\overrightarrow{0}}$ refinement is obtained. ${ }^{9}$

A complicating factor is that cortical somato- $\overrightarrow{\vec{\omega}}$ sensory evoked potentials are subject to attenuation by anaesthesia ${ }^{1011}$ and by hypotension. This $\vec{x}$ increases the difficulty of obtaining good quality: recordings in the operating theatre, which is never an? ideal electrophysiological environment.

Although somatosensory evoked potentials are $\vec{\circ}$

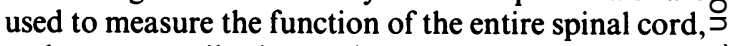
and some contribution to the potentials may be trans- $\vec{c}$ mitted by the anterior part of the spinal cord, the main pathway is via the dorsal columns. So far, in $\frac{2}{d}$ acute conditions - for example, in trauma, spinal operations, or vascular insults-the somatosensory $\vec{\varphi}$ evoked potential does seem to be a sensitive indicator of global spinal cord function. ${ }^{7}$ This is not necessarily. the case in chronic paraplegia.

Clearly, we do not yet have sufficient information to know whether, in man, paraplegia, or at least para-o paresis, may occasionally result when somatosensory $\frac{\mathcal{Q}}{\mathcal{Q}}$ evoked potentials have not been abolished. This willo be difficult to discover with the given incidence of $\overrightarrow{\vec{O}}$ neurological sequelae. The relationship of intra- 3 operative somatosensory evoked potential changes to the occurrence and severity of postoperative neurological sequelae has so far not been established. ${ }^{12} \rightleftharpoons$

In my inquiry I asked the 90 surgeons specifically응 about spinal cord monitoring using somatosensoryx evoked potentials. Of the 74 who replied, three $\frac{5}{3}$ surgeons only in the United Kingdom and Ireland were using this method and a surprising number had $O$ not heard of its existence.

\section{Need for bypass}

When digital palpation is used the blood supply will be considered inadequate when the distal aorta becomes slack after cross clamping; when electromanometric methods are used a mean pressure below $\omega$ $60 \mathrm{~mm} \mathrm{Hg}$ is usually considered an unsafe pressure with which to proceed with the operation withouto some form of bypass. Again, when somatosensory evoked potentials are used the disappearance of these ${ }^{-}$ potentials will indicate the need for some form of $\frac{0}{0}$ bypass.

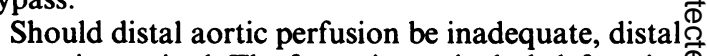
bypass is required. The forms in use include left atrio- $\stackrel{\mathbb{D}}{\Omega}$ femoral bypass, heparin bonded shunts, femoral veing 


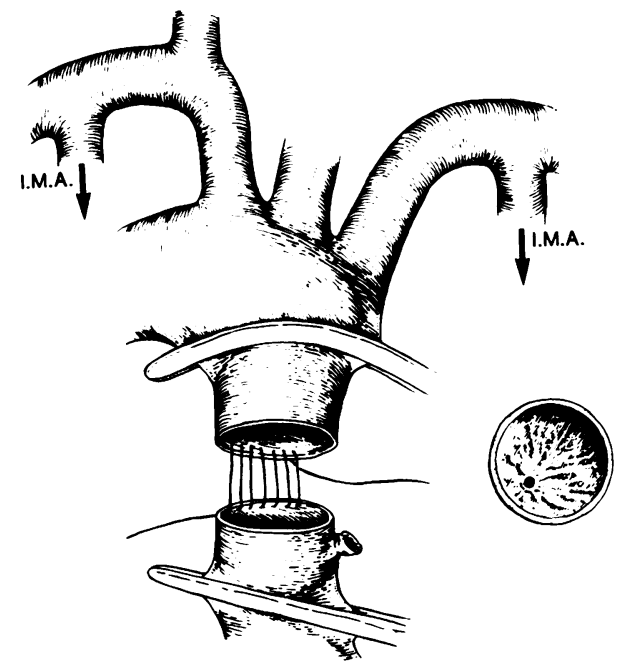

Fig 1 The usual type of coarctation, which is seen in the inset illustration to produce an almost total obstruction to flow. During the operation it is unnecessary to cross clamp the left subclavian artery and therefore bilateral collateral flow is ensured via the internal mammary and other branches of both subclavian arteries. IMA-internal mammary artery.

to femoral artery bypass, or surface cooling to $30^{\circ} \mathrm{C}$ with any of these forms of bypass. The exponents of very rapid aortic surgery ${ }^{1314}$ seem to manage with none of these aids (although their patients do not appear to be immune from paraplegia), but the surgeon of average ability is advised to err on the side of safety and use a bypass technique. ${ }^{15}$ We need to pay particular attention to those patients in whom

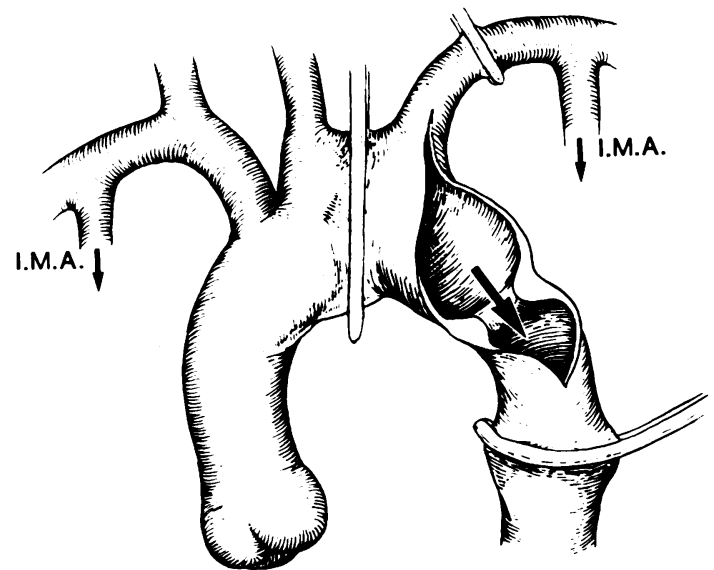

there is an appreciable blood flow through the coarctation, which will be lost to the lower aorta during aortic cross clamping.

\section{Anatomical variation and risk of paraplegia}

There are two main surgical varieties of coarctation. In the usual type the narrowing at the level of insertion of the ligamentum arteriosum into the aorta is an extremely tight and sometimes totally occluding diaphragm (fig 1). This is by far the most common variety and collaterals are obtained almost exclusively from the large branches of the subclavian arteries, which are the internal mammary and parascapular vessels. These patients present with classical signs, such as severe upper limb hypertension, absence of femoral pulsation, easily palpable collaterals over the shoulders and back of the chest wall, and well marked rib notching seen on the plain chest radiograph. In the second and rather less common variety the aorta has a longer and less severe stricture and total occlusion is rare. In this group of patients, apart from there being a variable and possibly inadequate collateral circulation provided by the branches of the subclavian arteries, very often a considerable amount of blood passes through the coarctation (fig 2a)

In the first variety cross clamping of the aorta above and below the coarctation does not interfere appreciably with distal blood flow. Furthermore, in these patients it is usually unnecessary to cross clamp the left subclavian to gain access, and the only interference with the lower anastomotic channels is by temporary clamping or ligation of one or two pairs of feeding intercostal arteries just proximal to the lower

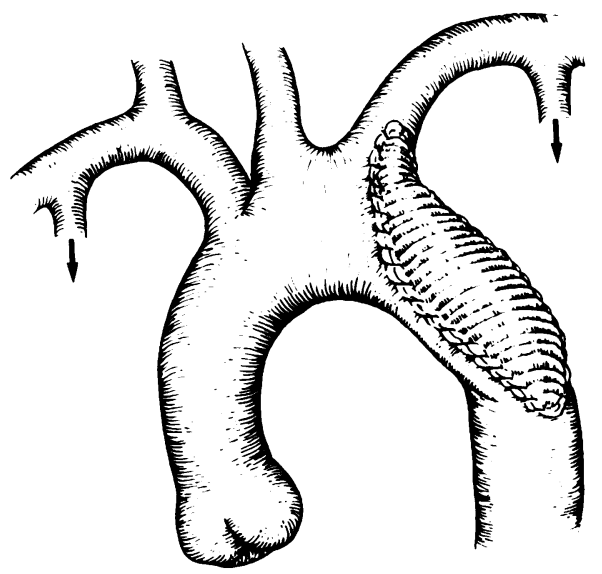

Fig 2 The less common type of coarctation. This produces less extreme narrowing and allows appreciable blood flow through the stenosis. When patch aortoplasty is undertaken the left subclavian artery has to be cross clamped. When this is done, the distal circulation is deprived not only of the significant flow through the stenosis but also of the blood supplied by the large branches of the left subclavian artery. IMA-internal mammary artery. 
clamp. On the other hand, in the second variety, where there is a long stricture, the operation of choice is often a patch annuloplasty: the stricture is incised longitudinally and the aorta widened by inserting a wide gusset of either prosthetic or biological material. To enable as large a gusset as possible to be inserted, the aorta usually has to be incised well proximally and up into the root of the left subclavian artery, which requires the left subclavian artery to be clamped throughout the procedure (fig $2 b$ ). In this group of patients therefore aortic cross clamping not only cuts off a perhaps appreciable blood flow that previously passed through the coarctation but also causes the loss of a most important contribution from the branches of the left subclavian artery during the procedure. Not surprisingly therefore, 10 of the 16 cases of paraplegia reported in this series occurred in patients with a long, narrow segment who underwent patch aortoplasty rather than resection and an end to end anastomosis. Given that this variety of coarctation is less common than is the complete or almost complete diaphragm and that aortoplasty is less common than resection with end to end anastomosis, these findings may well be significant.

In their review ${ }^{1}$ Brewer et al make no mention of the operation of patch aortoplasty. Nearly all the operations reported in his paper were undertaken in the earlier years of surgical correction and before the introduction of the operation of aortoplasty. Patch aortoplasty was introduced by Vosschulte in $1957^{16}$ but was not popularised until Cooley and his colleagues reported a substantial series of patients operated on from 1967 onwards. ${ }^{1718}$ In the report of Brewer et al great emphasis is placed on the number of intercostal arteries occluded and the duration of aortic occlusion, and their data support the widely held view that neither of these factors in itself is directly related to the development or otherwise of postoperative paraplegia. He did, however, make passing reference to the collateral circulation provided by the left subclavian artery, but stated that only five of more than 100 surgeons considered clamping of the left subclavian artery to be an important factor in the aetiology of paraplegia.

Our own findings therefore expose a further important incremental risk factor that has a bearing on this operation. During the first two decades of coarctation surgery the almost universal operation was resection and end to end anastomosis between occluding aortic clamps. When a long, narrow segment was present, this was excised; if it was then not possible to approximate the divided aortic ends a tubular graft had to be inserted. It was usually possible to place a Potts's spoon shaped clamp above the coarction in such a way as to enable good flow to be continued up the left subclavian artery. Although the tight, short coarctation still remains the most common form, patch annuloplasty is an increasingly fre- $\vec{F}$ quent operation nowadays. It is in many ways technically attractive as it does not require resection $\frac{\overline{0}}{0}$ of the aorta and, in children, it avoids the problem of $\overline{\bar{c}}$ failure of growth of an end to end anastomosis. The $\overrightarrow{\mathbb{D}}$ greater frequency of this operation may, however, $\stackrel{\Omega}{\Omega}$ have introduced an increased risk of paraplegia.

Occlusion of the left subclavian artery alone can $\vec{\circ}$ predispose to paraplegia, as one case reported to me in this series illustrates. This was a case of coarctation $\vec{\sigma}$ where the narrow segment was several centimetres $\vec{F}$ long, and it was decided to insert a bypass Dacron $\times$ graft from the left subclavian artery to the descending $\stackrel{t}{\mathrm{~T}}$ aorta without excising the coarctation or undertaking an aortoplasty. During this operation the left sub- $\vec{A}$ clavian artery was cross clamped throughout but the $\frac{\mathrm{S}}{-}$ distal aorta was at no time occluded, access for the lower anastomosis being provided by a side clamp. Nevertheless, this patient develped paraplegia.

\section{Future attitudes and prudent practice}

In the recent past, digital assessment of distal aortic. pressure could be accepted as providing an adequate standard of care during operations for coarctation of the aorta. With the development of accurate manometric methods, an increasing number of surgeons now use this technique-in 1985 more than half of the $\stackrel{\mathbb{Q}}{\AA}$ surgeons questioned did so. The conscientious use of $\overrightarrow{\vec{A}}$ either of these methods could in the past be regarded $\frac{3}{3}$ as adequate evidence of good practice. Eventually, however, the undertaking of aortic surgery without careful monitoring of spinal cord function might conceivably be regarded as negligent, especially if such an unmonitored procedure should be followed by neu- $\underset{x}{x}$ rological damage. There is certainly no guarantee that $\dot{\sigma}$ a distal pressure determined as adequate either by dig- 3 ital palpation or by electromanometric recording $\delta$ after cross clamping will prevent paraplegia. Even- $₹$ tually a more certain way of monitoring spinal cord 0 function, such as the evoking of somatosensory potentials, is likely to be available to all surgeons; but as yet these techniques are imperfect. My own experience indicates that whereas the majority of surgeons $\sigma$ practising these operations are unaware of somato- $N$ sensory evoked potential as a method of spinal cord $N$ monitoring, lawyers dealing with medical negligence $\bar{\sigma}$ claims are well aware of these developments and have asked whether spinal cord monitoring was used dur- $\frac{\overparen{D}}{\Phi}$ ing an operation on their paraplegic client. There is, $\stackrel{\oplus}{-}$ however, no firm evidence to sustain the view that the 0 absence of somatosensory evoked potential moni- $\bar{D}$

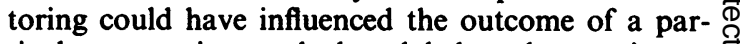
ticular operation and thus label such practise as $\mathbb{\otimes}$ negligent. The lawyers will invariably ask whether or 
not electromanometric methods rather than digital palpation were used to assess the adequacy of distal aortic pressure after cross clamping, and here they may be on firmer ground. Whereas in the past we could claim bad luck when patients developed paraplegia after an operation for coarctation, this may not be so in the future. It is therefore predicted that, if the reliability of the technique can be established, the use of spinal cord monitoring by the evoking of somatosensory potentials will be universally adopted. What then should be our attitude towards the monitoring of operations on the descending thoracic aorta?

I believe that the monitoring of spinal cord function by somatosensory evoked potentials may eventually be shown to be accurate and reliable, but this has not yet happened in the United Kingdom. On the other hand, the monitoring of distal aortic pressures by electromanometric methods is clearly more sensitive and accurate than is the assessment of digital palpation alone. Although many experienced and distinguished surgeons would disagree with this last sentiment and claim that in their hands digital aortic palpation has never been followed by paraplegia, it is likely that an uncomfortably large number of surgeons will have an operation complicated by paraplegia should they operate on a sufficient number of patients, whichever method of monitoring is used.

Cases are reported of paraplegia following short periods (under 20 minutes) of aortic cross clamping, confirming the view that in a very small number of patients even short periods of aortic cross clamping will not be tolerated-regardless of the skill and experience of the surgeon. It is advocated that sole reliance on estimation of aortic pressures by digital palpation should be abandoned and replaced in all cases by electromanometric measurement, to give our patients the most accurate spinal cord monitoring service universally available today, and to establish a new yardstick of acceptable care. Although the use of somatosensory evoked potential monitoring of the spinal cord is largely undeveloped in the United Kingdom, the work of Laschinger in the United States and Pollock in Glasgow shows clearly that this method has great potential. ${ }^{7-9}$

It is recommended that interested users, including thoracic surgeons, orthopaedic surgeons, and neurophysiologists collaborate and develop somatosensory evoked potential monitoring in their own departments, in the expectation that they will eventually have at their disposal a comprehensive and reliable system of spinal cord monitoring.

I am indebted to Dr Hilary Morgan of the Burden Neurological Institute, Bristol, for her review of this paper and to my many colleagues in cardiothoracic surgery who assisted with this survey.

\section{G KEEN \\ Bristol Royal Infirmary Bristol BS2 $8 \mathrm{HW}$}

\section{References}

1 Brewer LA, Fosburg RG, Mulder GA, Verska JJ. Spinal cord complications following surgery for coarctation of the aorta. A study of 66 cases. J Thorac Cardiovasc Surg 1972;3:368-81.

2 Adamkiewicz A. Die Blutgefasse des Mensch Lichen Ruckenmarkes. I Teil. Die Gefasse der Ruckenmarksubstanz. II Teil. Die der Ruckenmarkoberflache. Sitz Akad Wiss Wein Math Natur Klass 1882;84:469 and 1882:85:101-30.

3 Svenson LG, Rickards E, Coull A, Rogers G, Fimmel J, Hinder RA. Relationship of spinal cord blood flow to vascular anatomy during thoracic aortic cross clamping and shunting. J Thorac Cardiovasc Surg 1986;91:71-8.

4 Domisse GF. The blood supply of the spinal cord. $J$ Bone Joint Surg 1974;56B:225-35.

5 Wadouh F, Arndt CF, Metzger H. Hartmann M, Wadouh R, Borst HG. Direct measurements of oxygen tension on the spinal cord surface of pigs after occlusion of the descending aorta. $J$ Thorac Cardiovasc Surg 1985;89:787-94.

6 Hughes RK, Reemtsma K. Correction of coarctation of the aorta: manometric determination of safety during test occlusion. J Thorac Cardiovasc Surg 1971;62:31-7.

7 Laschinger JC, Cunningham JN jun, Catinella FP, et al. Detection and prevention of intraoperative spinal cord ischemia after cross clamping of the thoracic aorta: use of somatosensory evoked potentials. Surgery 1982;92:1109-14.

8 Laschinger JC, Cunningham JN jun, Nathan IM, Knopp EA, Cooper MM, Spencer FC. Experimental and clinical assessment of the adequacy of partial by-pass in maintenance of spinal cord blood flow during operations on the thoracic aorta. Ann Thorac Surg 1983;36:417-26.

9 Pollock JC, Jamieson MP, McWilliam R. Somatosensory evoked potentials in the detection of spinal cord ischaemia in aortic coarctation repair. Ann Thorac Surg 1986;41:251-4.

10 Clark DL, Rosner BS. Neurophysiological effects of general anaesthesia. I. The electroencephalogram and sensory evoked responses in man. Anaesthesiology 1973;38:564-82.

11 Allison T, Goff WR, Abrahamian HA, Rosner BS. The effects of barbiturate anaesthesia upon human somatosensory evoked responses. Electroenceph Clin Neurophysiol 1963;24 (suppl):68-75.

12 Mizrahi EM, Crawford ES. Somatosensory evoked potentials during reversible spinal cord ischaemia in man. Electroenceph Clin Neurophysiol 1984;58:120-6.

13 Crawford ES, Fenstermacher JM, Richardson W, Sandiford F. Reappraisal of adjuncts to avoid ischemia in the treatment of thoracic aneurysms. Surgery 
1970;67:182-9.

14 Najafi H, Javid H, Hunter J, et al. Descending aortic aneurysmectomy without adjuncts to avoid ischemia. Ann Thorac Surg 1980;30:326-37.

15 Katz NM, Blackstone EH, Kirklin JW, Karp RB. Incremental risk factors for spinal cord injury following operation for acute traumatic aortic transection. $J$ Thorac Cardiovasc Surg 1981;81:669-74.

16 Vosschulte K. Isthmusplastic zur behandlung der aortem isthmusstenose. Thoraxchirurgie 1957;4:443.

17 Reul GJ, Kabbani SS, Sandiford FM, Wukasch DC Cooley DA. Repair of coarctation of the thoracic aorta by patch aortoplasty. $J$ Thorac Cardiovasc Surg 1974;68:696-714.

18 Hesslein PS, McNamara DG, Morrish MJ, Hallman GL, Cooley DA. Comparison of resection versus patch aortoplasty for repair of coarctation in infants and children. Circulation 1981;64:164-73. 